\title{
Heavy Ion Composition of Mercury's Magnetosphere
}

\author{
P. Wurz ${ }^{1}$, D. Gamborino ${ }^{1}$, A. Vorburger ${ }^{1}$, and J. M. Raines ${ }^{2}$ \\ ${ }^{1}$ Physics Institute, University of Bern, Bern, Switzerland.
${ }^{2}$ Dept. of Climate and Space Sciences and Engineering, University of Michigan, Ann Arbor, Michigan, \\ USA.
}

\section{Key Points:}

- Calculated Mercury's exosphere chemical composition from first principles

- Calculated Mercury's magnetosphere ion input from neutral exosphere and compared this to MESSENGER ion in situ data

- Calculated new upper limits for the volatile species in Mercury's exosphere

This is the author manuscript accepted for publication and has undergone full peer review but has not been through the copyediting, typesetting, pagination and proofreading process, which

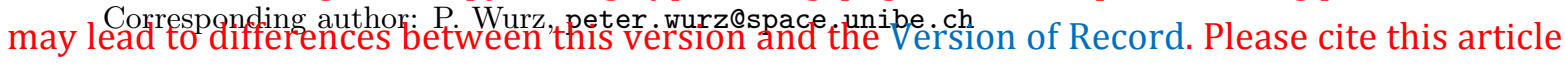
as doi: 10.1029/2018JA026319 


\begin{abstract}
We modeled the exospheric densities for sputtering and thermal desorption in detail for the time period of the first MESSENGER flyby of Mercury. From the exospheric densities we calculate ion production rates. These ions will be transported to the location of MESSENGER if they are produced on magnetic fields lines connecting the cusp with the downwind side. From these ions we produce mass spectra that we compared with the Fast Imaging Plasma Spectrometer (FIPS) measurements performed during this flyby. We find good qualitative agreement between the modeled and the measured ion mass spectrum. We find that sputtering is a major process to contribute to the population of planetary ions in the magnetosphere because of the large scale height of the exospheric particles, and the resulting long flight times. In addition, thermal desorption of $\mathrm{Na}$ contributes significant amounts to the magnetospheric ion population. From the volatile species we can identify $\mathrm{He}, \mathrm{OH}, \mathrm{H}_{2} \mathrm{O}$, and $\mathrm{Ne}$ in the measured mass spectrum. However, for most of the volatile species the reported upper limits must be reduced by $2-3$ orders of magnitude to be compatible to the measured ion spectrum.
\end{abstract}

\title{
1 Introduction
}

The atmosphere of planet Mercury, a thin gaseous envelope, is the result of several external agents acting on the surface. This atmosphere is a good example of a surface-bound exosphere, where all its material has its direct origin on the surface of the planet. It has been reviewed recently by Killen et al. (2007). There are volatile species released thermally or by photon-stimulated desorption from the illuminated hermean surface (Killen \& Ip, 1999; Wurz \& Lammer, 2003). Moreover, at places where the solar wind (Kallio \& Janhunen, 2003) or magnetospheric ions (Delcourt et al., 2003) hit the surface, atoms and molecules are sputtered from the surface and thus refractory species also become part of the hermean exosphere, having larger scale heights than the thermally released species (Wurz et al., 2010). Finally, also micro-meteorite impacts release material from the surface into the exosphere.

Mercury's dipole magnetic field is small (Anderson et al., 2011) thus the size of Mercury's magnetosphere is very small, with the distance of the bow-shock from Mercury's center in the upwind direction being only about $1.3 R_{M}$, with $R_{M}$ the radius of Mercury. Since Mercury does not have an ionosphere, there is almost no inertia in the magnetospheric current system and the magnetosphere quickly changes with changing solar wind conditions (Raines et al., 2015).

Solar wind plasma enters the hermean magnetosphere and dominates the magnetospheric plasma. Computer modelling of Mercury's magnetosphere showed that large fractions of the magnetosphere are open to the solar wind plasma around the cusps where solar wind ions can access the surface. These large open fractions predicted by many hermean magnetospheric models are rare occurrences in MESSENGER observations. However, MESSENGER observations clearly show that solar wind plasma is present in the cusp on nearly every orbit, and that precipitating fluxes, while highly variable, are substantial on a regular basis (Raines, Gershman, et al., 2014; Raines, Tracy, et al., 2014). The size and exact location of these open areas depend on the solar wind plasma parameters, foremost the speed, the density, and the magnetic field (Kallio \& Janhunen, 2003; Massetti et al., 2003). These calculations predict an integrated ion flux onto Mercury's surface in the range of $1.1 \cdot 10^{25}-3 \cdot 10^{26} \mathrm{~s}^{-1}$ depending on solar wind plasma parameters and the interplanetary magnetic field. Most of the ion precipitation is at longitudes within $\pm 60^{\circ}$ from the subsolar point at latitudes between $40^{\circ}$ and $60^{\circ}$. In addition, there is a narrow band of magnetospheric ions precipitating onto the surface all around the planet at mid latitudes, the so-called auroral precipitation (Delcourt et al., 2003; Kallio \& 
Janhunen, 2003; Massetti et al., 2003), where the proton fluxes are of the order of $10^{11} \mathrm{~m}^{-2} \mathrm{~s}^{-1}$.

The first six months of orbital measurements by the MESSENGER magnetometer indicate a mean latitudinal extent of the northern cusp of $\sim 11^{\circ}$. From the deficit of the average magnetic pressure in the cusp, Winslow et al. (2012) estimate that $(1.1 \pm 0.6) \times 10^{24}$ protons s ${ }^{-1}$ bombard the surface over an area of $(5.2$ $\pm 1.6) \times 10^{11} \mathrm{~m}^{2}$ near the northern cusp centered at $74.7^{\circ}$ Mercury Solar Orbital (MSO) latitude on the surface. This gives a solar wind flux of $2.11 \times 10^{12} \mathrm{~m}^{-2} \mathrm{~s}^{-1}$ onto the surface, which agrees with earlier estimates within a factor 2 (Kallio \& Janhunen, 2003; Massetti et al., 2003). The total particle flux to the surface near the southern cusp is predicted to be a factor of 4 larger than in the north. The northern cusp is clearly evident during IMF conditions but, on average, exhibits $40 \%$ higher plasma pressures during anti-sunward conditions (Winslow et al., 2012), indicating that the effect of IMF $B_{x}$ direction is present (Massetti et al., 2003; Winslow et al., 2012). The latitude of the southern cusp is at $64^{\circ} \mathrm{S}$. Mercury's magnetic field model predicts a surface field strength at this latitude of $158 \mathrm{nT}$ (Anderson et al., 2011). From the ratio of the surface field strength in the south to that in the north the authors estimate that the cusp area in the south extends over $2 \times 10^{12} \mathrm{~m}^{2}$. And the number of particles reaching the surface in the southern cusp region is correspondingly higher, $4 \times 10^{24}$ particles $\mathrm{s}^{-1}$ (Winslow et al., 2012). For the northern and the southern cusp the ion precipitation evaluates to about the same flux of about $2 \cdot 10^{12} \mathrm{~m}^{-2} \mathrm{~s}^{-1}$. Thus, we use a precipitating solar wind flux of $2 \cdot 10^{12} \mathrm{~m}^{-2} \mathrm{~s}^{-1}$ in our modelling of the sputtered fluxes. This is about half the flux we used earlier (Wurz et al., 2010), based on estimates available at that time (Massetti et al., 2003).

Because Mercury is very close to the Sun, photoionization of exospheric species will contribute to the magnetospheric ion population. Ionized exospheric atoms become part of Mercury's magnetosphere, will be transported from their origin on the dayside, especially from the cusp region, to equatorial latitudes at the downwind side of the magnetosphere within a few minutes (Delcourt, Moore, Orsini, Milillo, \& Sauvaud, 2002), and some of the ions will return to Mercury's surface and impact at the auroral precipitation bands. Depending on the amount and the mass of these photo-ions, they may alter Mercury's magnetospheric dynamics. $\mathrm{Na}^{+}$ions have been studied in detail in this respect. For example, the complex fate of $\mathrm{Na}^{+}$ ions in the magnetosphere, their transport, energization, and loss has been studied in detail by Delcourt et al. (2003), who find that a fraction of these $\mathrm{Na}^{+}$ions are accelerated back towards the surface to precipitation regions at mid-latitudes of about $30^{\circ}-40^{\circ}$ over a wide range of longitudes with ion fluxes in the range of $10^{5}$ $10^{6} \mathrm{~cm}^{-2} \mathrm{~s}^{-1}$.

\section{Observations}

Mass spectra of magnetospheric ions where recorded with the Fast Imaging Plasma Spectrometer (FIPS) instrument of the Energetic Particle and Plasma Spectrometer (EPPS) on the MESSENGER spacecraft during its first flyby on 14 January 2008, 19:04:39 UTC (Zurbuchen et al., 2008). FIPS is designed to characterize ionized species in a range of energy-per-charge $(E / Q)$ from $<50 \mathrm{eV} / \mathrm{Q}$ up to 13 $\mathrm{keV} / \mathrm{Q}$ with a novel electrostatic analyzer (ESA) system geometry that enables a large instantaneous field-of-view of about $1.4 \pi$ steradians (Andrews et al., 2007). The plasma parameters for Mercury cannot be fully characterized by FIPS because of the limited field-of-view of the instrument being located behind the heat shield of the spacecraft. 


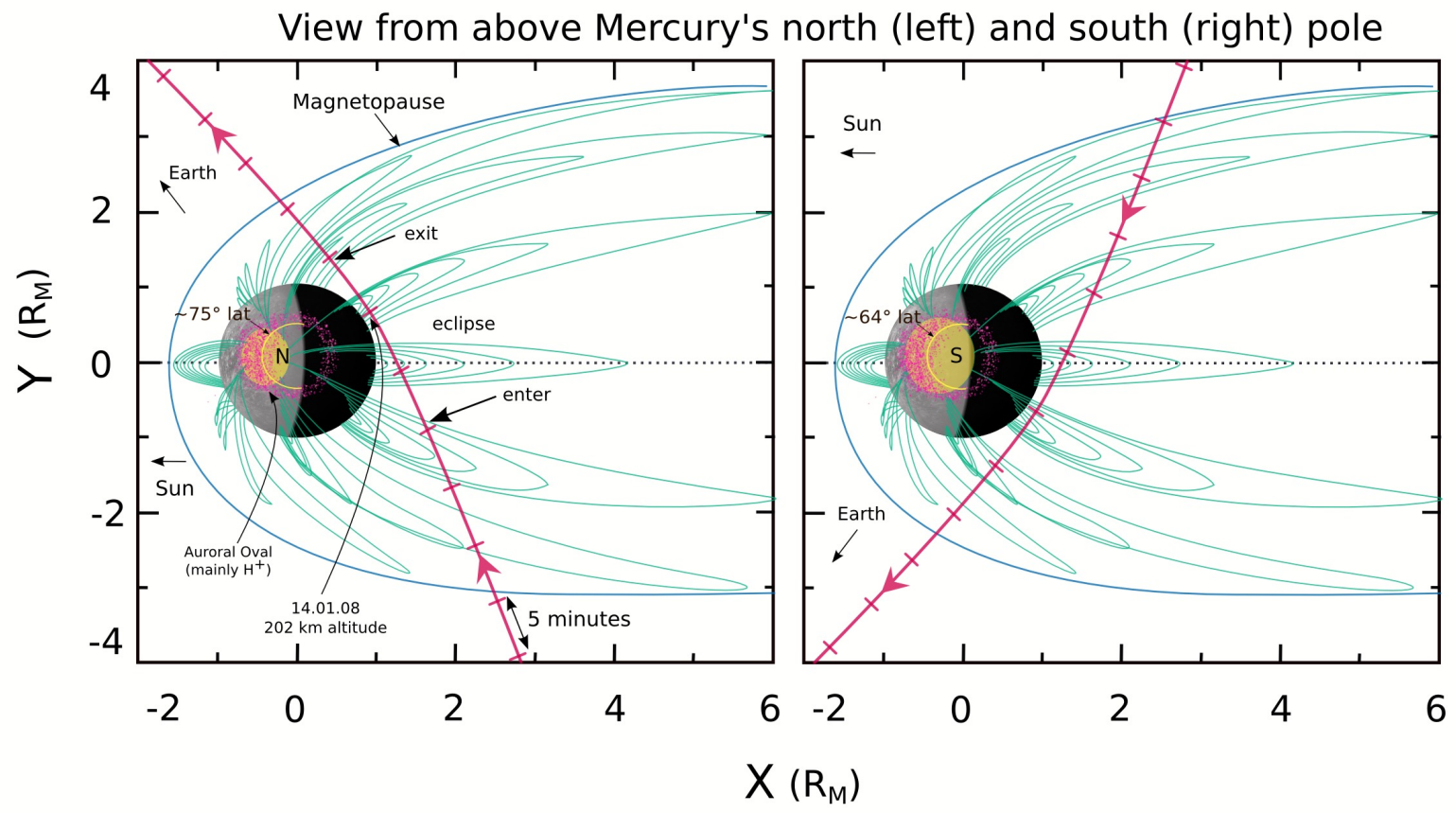

Figure 1. Mercury's magnetic field lines representation as seen from above and from below the planet, which were take from an image from NASA/Johns Hopkins University Applied Physics Laboratory/Carnegie Institution of Washington. Pink lines show the trajectory of the first MESSENGER flyby of Mercury (Source of http://messenger.jhuapl.edu/), tick marks indicate 5 minute time intervals. Yellow cusp regions are based on Winslow et al. (2012), pink dots roughly illustrate areas where ions are produced.

Since FIPS is a plasma composition analyzer, its mass resolution of about $m / \Delta m \approx$ 10 is sufficient for the plasma science investigations, but somewhat low for detailed chemical composition analysis. Therefore, Zurbuchen et al. (2008) presented the composition analysis of their measurements restricted only to mass-groups, like the water group (with the ion species $\mathrm{O}^{+}, \mathrm{OH}^{+}, \mathrm{H}_{2} \mathrm{O}^{+}$, and others), the Na-group $\left(\mathrm{Na}^{+}\right.$ and $\left.\mathrm{Mg}^{+}\right)$, the Si-group $\left(\mathrm{Al}^{+}, \mathrm{Si}^{+}, \mathrm{S}^{+}\right)$and others.

The geometry of the first flyby is shown in Fig. 1 together with a representation of the magnetosphere. The flyby was on the anti-sunward side of Mercury with the closest approach at $37.730^{\circ} \mathrm{E}$ longitude near Mercury's equator at $4.521^{\circ} \mathrm{S}$ at a minimum distance of $201.7 \mathrm{~km}$ from the surface.

The Fast Imaging Plasma Spectrometer (FIPS) instrument on the MESSENGER spacecraft measured a mass-per-charge $(\mathrm{m} / \mathrm{q})$ spectrum of ions with $3.8<$ $m / q<42$ accumulated in Mercury's magnetosphere between 18:43 and 19:14 UTC during the first flyby on 14 January 2008 (upper panel in Figure 2) (Zurbuchen et al., 2008), when the spacecraft was passing Mercury on the downwind side. These data are shown in Fig. 2 in the upper panel where the different mass groups are apparent. Since FIPS was operated in a different mode during later times of the mission, no further ion mass spectra are available.

Following the first flyby, onboard classification of ions by $m / q$ was eliminated from the FIPS flight software so that most of the telemetry could be devoted to full 
event (pulse-height analyzed, PHA) words. The assignment of these event words to ion groups on the ground proceeds using a different procedure (Raines et al., 2013) so that $m / q$ spectra are no longer produced. A new algorithm for assigning counts to ion groups is being developed (Tracy, 2016), which can correct for overlap between ion groups in FIPS TOF measurements. This algorithm can produce $m / q$ spectra comparable to those from the first flyby, though at much higher fidelity, so that $m / q$ spectra from orbital data should be available in the future.

We will interpret the measured ion composition spectrum by FIPS with the help of our Mercury exosphere model (Wurz \& Lammer, 2003; Wurz et al., 2010) because the details of the ion composition are an important input for the interpretation of the magnetospheric plasma data, and its modeling. Since the sputtered exospheric particles originate directly from the surface, the comparison between the measured ion composition and modeled one will provide clues about the surface composition. Similarly, the abundance of volatile species in the exosphere can be constrained by the comparison between the measured and modeled ion composition.

\section{Modeling}

To predict the ion composition we use our exosphere model, which was described in detail earlier (Wurz \& Lammer, 2003; Wurz et al., 2007, 2010). There are four processes of particle release from the surface implemented in the model: thermal release (TD), ion-induced sputtering (SP), micro-meteorite impact vaporization (MIV), and photon-stimulated desorption (PSD). For the most recent formulation of PSD see Gamborino and Wurz (2018). The released particle flux from the surface is calculated from the action of an external driver (e.g. ion precipitation to the surface for SP) and the surface composition. The angular and energy distributions for each release process are implemented as three-dimensional velocity distributions and evaluated by the Monte Carlo (MC) method using $10^{6}$ to $10^{7}$ particles. The resulting elliptic or hyperbolic trajectories of the released particles are calculated analytically in the plane of their movement (Vorburger \& Wurz, 2018), until the particle falls back onto the surface, leaves the calculation domain, or is ionized and is carried away be the electro-magnetic fields of Mercury's magnetosphere. We use an average latitude of $50^{\circ}$ and a longitude of $0^{\circ}$, the center of the cusp region, for our calculations. For the sputtered flux we use an ion precipitation of $2 \cdot 10^{12} \mathrm{~m}^{-2} \mathrm{~s}^{-1}$, as discussed above, and for the thermal release we use the local surface temperature of $550 \mathrm{~K}$ calculated for this location and Mercury's orbital position. From the ensemble of trajectories we derive various quantities for the exosphere, of which the radial density profile of neutral particles and the ion production are used for the present studies.

Ionization along a particle trajectory is calculated using the solar ionization rates given at the SwRI photoionization web page (http://phidrates.space.swri.edu). The MC code keeps track of the ion production in the exosphere and from that calculates an ion flux into the magnetosphere. The transport of ions inside the magnetosphere is beyond the possibilities of our MC code, but is based on theoretical considerations detailed below. Since the ion production is at the cusp on the dayside and the MESSENGER observations were on the downwind side only qualitative comparisons between the measurements and the model can be performed. There is no spatial detail of the ion composition measurements available since the accumulated FIPS mass spectrum is integrated over the magnetosphere part of the flyby trajectory (Zurbuchen et al., 2008). Thus, using one-dimensional radial density profiles for the neutral exosphere densities and the ion production is sufficient for this comparison.

When a particle is ionized it becomes part of the magnetospheric particle population if the ion is formed at an altitude below the magnetopause, the boundary 
that separates the magnetosphere from the solar wind flowing past it. The altitude of this boundary in area around the cusp varies, most often falling within the range of 1000-2000 km (Winslow et al., 2012), thus we use $1500 \mathrm{~km}$ altitude for this discrimination (see Fig. 1). The majority of ions created outside the magnetopause are carried away with the solar wind as it flows past the magnetosphere. Motion of ions due to the electric and magnetic fields of this system and their transport to the location of MESSENGER are not modeled here. It is assumed that this acceleration and transport does not discriminate significantly in mass. Delcourt (2013) showed in recent modelling that because of the abrupt energization of newly-formed ions in the hermean magnetosphere, which is parallel to the magnetic field line, the daysideto-nightside transport depends little upon the ion mass. This finding is in agreement with later MESSENGER FIPS measurements, which exhibit qualitatively similar features for both $\mathrm{Na}^{+}$-group and $\mathrm{O}^{+}$-group ions (Raines et al., 2013). Because the ion pickup and acceleration has an unknown efficiency for the transport of planetary ions from the dayside to the nightside, and the field-of-view limitations of FIPS, only qualitative comparisons between the observations and the model will be performed.

Ion transport and acceleration has been studied theoretically in detail recently (Delcourt, 2013; Delcourt et al., 2002). These authors find that the two major contributions to ion acceleration from the dayside to the tail result from the curvature of the magnetic field lines and from the curvature of the $\mathbf{E} \times \mathbf{B}$ drift paths. Both accelerations are large because of the small magnetosphere of Mercury and the associated strong curvature of the magnetic field lines. This leads to a strong centrifugal acceleration of ions tailward from high to low latitudes, so that these ions enter the central plasma sheet on the downwind side in Mercury's magnetosphere. Depending on the cross-polar cap potential drop, this acceleration results in planetary ion energies ranging from hundreds of $\mathrm{eV}$ up to over $10 \mathrm{keV}$ at their arrival at the nightside equatorial region (Delcourt, 2013). These energies are attained independent of the initial ion energy at their formation, even when the initial energy is well below the escape energy. Based on this tailward acceleration, we assume that the planetary ions recorded by FIPS will be well inside its energy range. Actually, later FIPS measurements reported an average energy of $2-4 \mathrm{keV}$ for the recorded planetary ions, with substantial fractions of the $\mathrm{Na}^{+}$-group population at energies up to $10 \mathrm{keV}$ (Raines et al., 2013).

Most of the ions causing sputtering of particles are from the solar wind hitting the surface at the cusps located at mid latitudes (Winslow et al., 2012). The plume of released particles is much bigger than the cusp area and trajectories of many exospheric particles are in areas of magnetospheric field lines connecting to the tail.

The original composition model of Mercury's surface (Wurz et al., 2010) was updated by comparison with the surface composition data that later became available by the MESSENGER investigations, as discussed recently (Pfleger et al., 2015). For this work we also considered the most recent results from MESSENGER (Peplowski et al., 2016, 2015).

Sputtering and micro-meteorite impact promote similar quantities of neutral particles into the exosphere (Wurz et al., 2010). The scale heights of sputtered particles are significantly larger than for MIV (Wurz et al., 2010), thus having longer flight times in the exosphere. This results in larger photoionization yields from SP, thus their contribution to the ion input to the magnetosphere is dominating over the MIV produced ions. Therefore, we consider only sputtering for the contribution of refractory material in the comparison with the FIPS data.

Thermal desorption and photon-stimulated desorption promote volatile species into the exosphere. For most of the volatile species only the TD process applies. For $\mathrm{Na}$ and K, both TD and PSD are possible release processes. At the mid latitudes 
considered here for the cusp region, the surface temperature is about $550 \mathrm{~K}$ resulting in very high evaporation rates for $\mathrm{Na}$ and $\mathrm{K}$ from the population that is physically adsorbed on the surface. Theoretical evaporation fluxes are $1.2 \cdot 10^{23} \mathrm{~m}^{-2} \mathrm{~s}^{-1}$ and $2.4 \cdot 10^{24} \mathrm{~m}^{-2} \mathrm{~s}^{-1}$ derived from the $\mathrm{Na}$ and $\mathrm{K}$ vapor pressures, respectively (Lide, 2003), which means that all $\mathrm{Na}$ and $\mathrm{K}$ on the surface is immediately released to the exosphere, and the released flux is limited by the availability of $\mathrm{Na}$ and $\mathrm{K}$ on the surface. Since the release by PSD is many orders of magnitudes less efficient than thermal desorption in releasing $\mathrm{Na}$ and $\mathrm{K}$ atoms from the population that is physically adsorbed by the surface, all the Na and K is released thermally, and PSD is therefore not considered further.

To allow for a qualitative comparison of the measured ion composition spectrum by FIPS with the prediction of our model we simulated the FIPS instrument performance where we assumed that the width of a mass peak in the FIPS spectrum is proportional to the mass of the species, with the scaling of the mass resolution derived from the end points in the measured mass spectrum given by the peak at mass 4 and mass 40. This assumption shows good agreement for the peak shapes when comparing the measured and modeled spectra. Relative sensitivities for all species where estimated, again, based on a carbon-foil time-of-flight mass spectrometer (Wurz, 1999).

\section{Results}

The exosphere model provides density profiles for the entire exosphere altitude range. Table 1 shows the detailed results of the calculation for the refractory species sputtered from the surface, using the solar wind flux of $2.1 \times 10^{12} \mathrm{~m}^{-2} \mathrm{~s}^{-1}$, see above, during the flyby, and photoionization in the exosphere. The calculation is based on the global chemical composition of Mercury's surface given before (Wurz et al., 2010) with updates based on recent MESSENGER data (Peplowski et al., 2016, 2015). As example, exospheric density values for the sputtered species at the surface and the released flux are given in Table 1. Ionization fractions are calculated along the trajectories of the released particles based on the photoionization rate. In the absence of electro-magnetic fields, the ionization fraction times the neutral density gives an exospheric ion density. Here we calculate the ion fluxes introduced into the magnetosphere from the neutral fluxes released from the surface. For the whole exospheric density profile $\epsilon_{i, \infty}$ is the fraction of the produced ions to the neutral particle flux integrated over altitude from the surface to infinity. The fraction $\epsilon_{i, 1500}$ is where the integral extends only to $1500 \mathrm{~km}$ altitude, and these ions will become part of the magnetospheric ion population. Finally, Table 1 gives the fluxes of ions entering the magnetospheric system. Figure 3, left panel, shows the density profiles for refractory species released by solar wind sputtering from the surface. Sputtered particles have large exospheric scale heights, about $1000 \mathrm{~km}$, because of the energy imparted to sputtered atoms (Wurz et al., 2010). Nevertheless, most of the ion production is near the surface, which makes the choice of $1500 \mathrm{~km}$ for the boundary of the magnetosphere very uncritical.

Table 2 shows the detailed results of the calculation of thermally released volatile species in the same format as Table 1, and Figure 3, right panel, shows the density profiles for volatile species based on the compilation by Killen and Ip (1999). Note that for most of the volatile species only upper limits of their exospheric densities are available. $\mathrm{Na}$ and $\mathrm{K}$ are also thermally released from the population that is physically adsorbed by the surface, in contrast to sputtering and MIV that release $\mathrm{Na}$ and $\mathrm{K}$ species from the minerals present on the surface (reported in Table 1). Since the scale heights for thermally released particles are much lower than for sputtering, there is a significantly lower production of photo-ions in the exosphere from thermally released particles. Nevertheless, some species would provide ion input into 


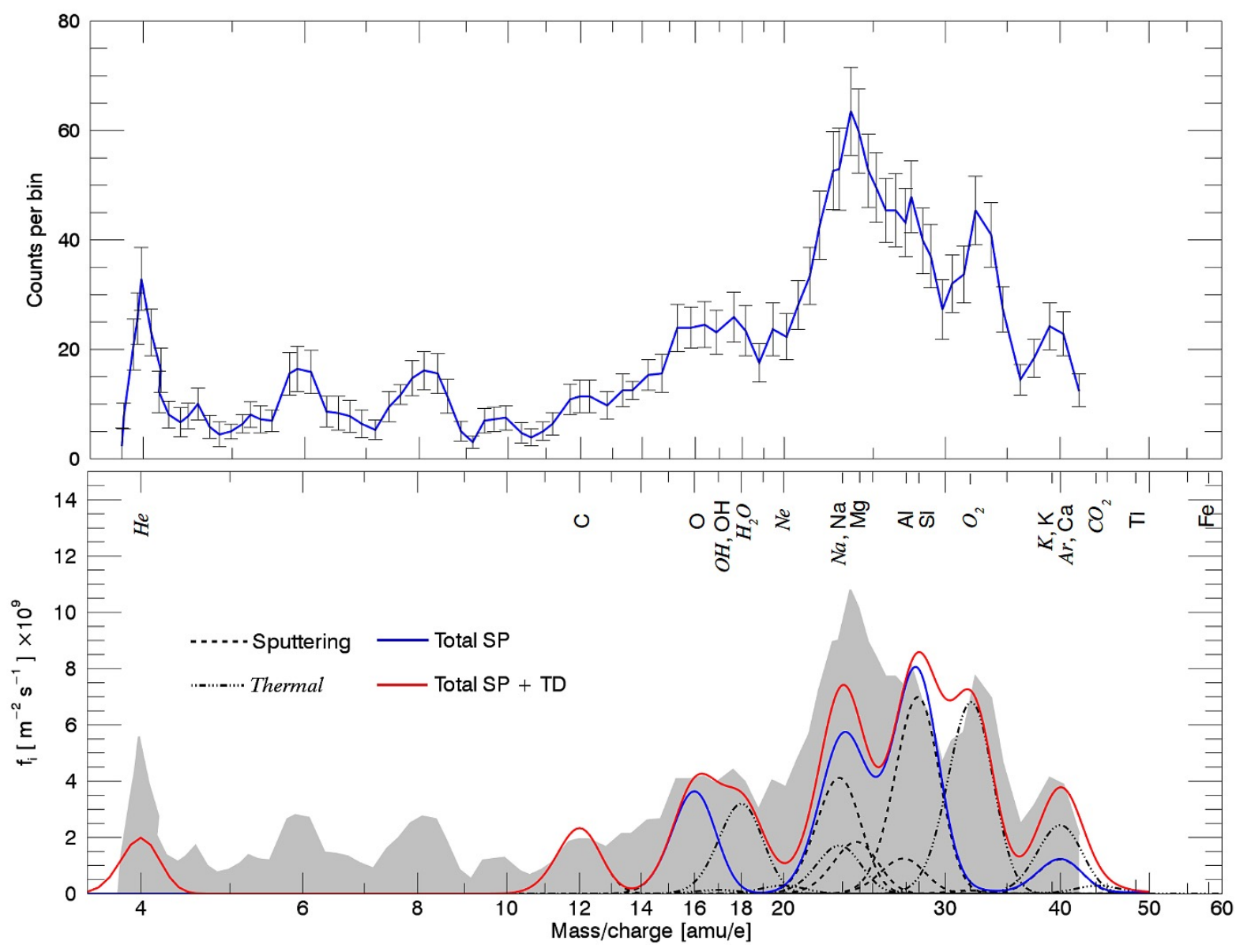

Figure 2. Upper panel: Measured ion composition data from MESSENGER FIPS at the downwind side of Mercury (Zurbuchen et al., 2008) during flyby (see Fig. 1). Lower panel: Calculated ion fluxes from the exosphere model with modeled mass resolution for FIPS. Black lines show the individual contributions from the different ions where Gaussian peak shapes are used with parameters from the observations. The blue line shows the sum of all mass peaks from sputtering, the red line shows the sum of all mass peaks. 

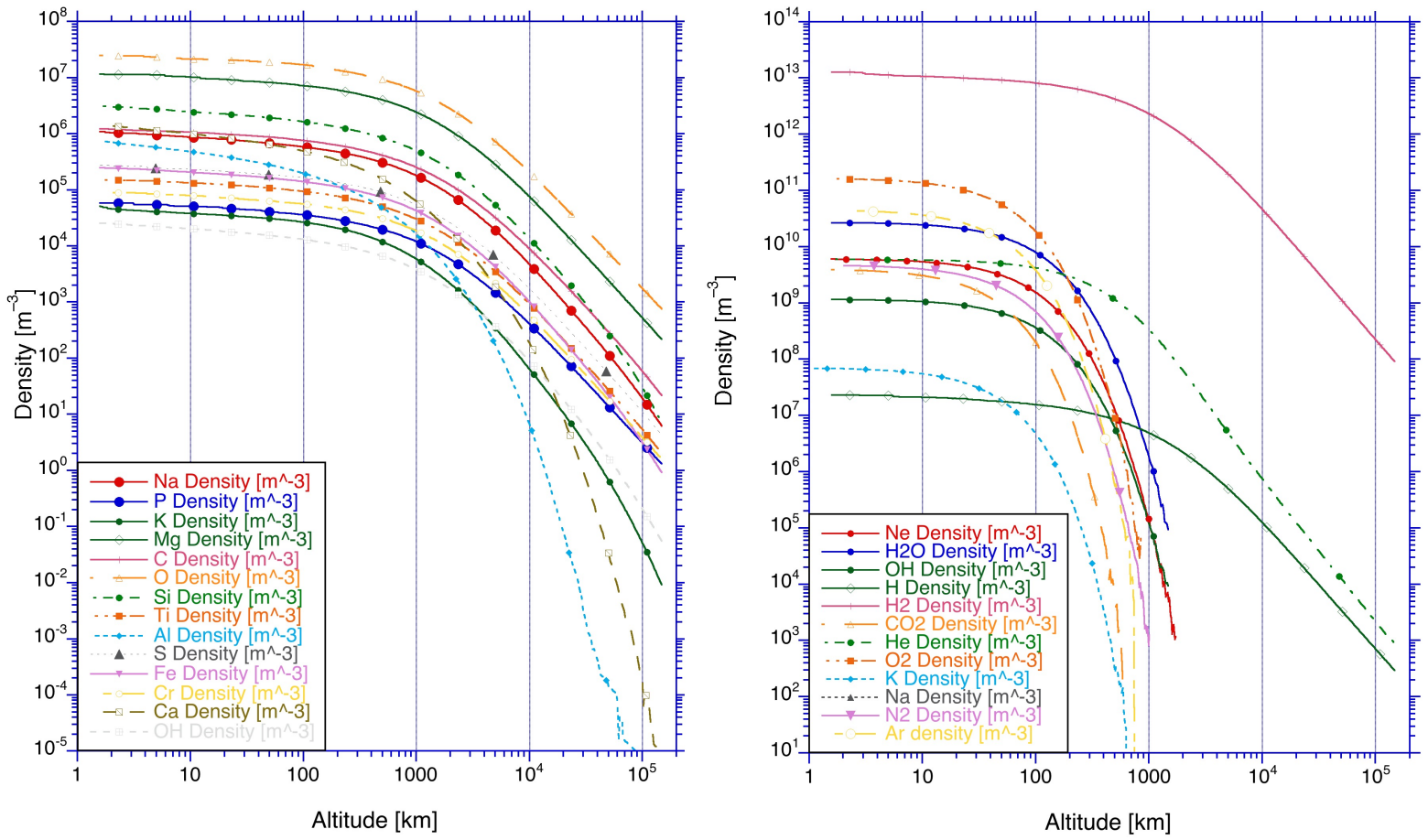

Figure 3. Left: Calculated density profiles for refractory species from solar wind sputtering. Exospheric surface densities are taken from (Wurz et al., 2010), additive model, with updates based on (Peplowski et al., 2016, 2015). Right: Calculated density profiles for volatile species by thermal release. Exospheric surface densities are taken from (Killen \& Ip, 1999), or are revised based on comparisons with the FIPS spectrum (see main text), and are mostly upper limits. $\mathrm{Na}$ and $\mathrm{K}$ are from volatile release, based on their calculated surface abundance. Note that the vertical scales are different for the left and right panel. 
the magnetosphere that is much more than is compatible with the FIPS observations.

The lower panel in Fig. 2 shows the modeled mass spectrum for sputtered and thermally released particles, respectively, based on the ion fluxes entering the magnetosphere. For the sputtered ions considered in the model, we find a fair agreement between the measured and the modeled mass spectra. The situation is different for the volatile species (Table 2), where the reported upper limits (Killen \& Ip, 1999) have to be reduced considerably to be compatible with the FIPS spectrum. This will be discussed in detail below.

At the lowest masses, the volatile species $\mathrm{H}$ and $\mathrm{H}_{2}$ are outside the measurement range of the reported FIPS $m / q$ spectrum. The first species to be compared is He at mass 4, for which an exospheric density of $6.0 \times 10^{9} \mathrm{~m}^{-3}$ has been determined (see review by Killen and Ip (1999)), and which is used in the model. The modeled He is compatible with the measured ion mass spectrum. If He is from solar wind implantation into the surface regolith we can calculate its exospheric density assuming that the regolith is saturated with He, i.e., the implantation rate and the release rate from the regolith are the same. From that assumption we get a He exospheric density at the surface of $7.27 \times 10^{9} \mathrm{~m}^{-3}$, which is in reasonable agreement with the published value and the FIPS measurement. This estimate considers also the accumulation of He in the hermean exosphere, similar to the noble gases in the exosphere of the Moon (Wurz, Abplanalp, Tulej, \& Lammer, 2012).

Double charged ions are clearly present in the measured ion mass spectrum at $m / q=6$ and at $m / q=8$, see Fig. 2 upper panel, likely being $\mathrm{C}^{2+}$ and $\mathrm{O}^{2+}$ ions, and possibly $\mathrm{Si}^{2+}$ at $m / q=14$. In our model we do not calculate the formation of doubly ionized species. We note however, that the photoionization rates to form them are about a factor 100 less than for the formation singly charged ions. The transport time of a few minutes to the tail (Delcourt, 2013; Delcourt et al., 2003) is not sufficient to produce significant amounts of doubly ionized species via photoionization. Thus, another mechanism, perhaps charge exchange or electron ionization might be responsible for their formation.

At $m / q=12$ we have the first ion resulting from sputtering, carbon, in good agreement with the measurements.

At the water group, at $m / q=16 \ldots 20$, we have only the contribution of $\mathrm{O}^{+}$ from sputtering, which is in good agreement with the FIPS spectrum. The $\mathrm{OH}^{+}$ contribution from sputtering is negligible. Other ion contributions to this mass group must arise from ionized volatile species in the exosphere. The $\mathrm{OH}$ contribution from the volatile inventory is compatible with the measured mass spectrum, thus its exospheric density should be close to $n_{0}(\mathrm{OH})=1.4 \times 10^{9} \mathrm{~m}^{-3}$ at the surface. The $\mathrm{H}_{2} \mathrm{O}$ from the volatile inventory is not compatible with the mass spectrum, its exospheric density should be much lower than the reported upper limit of $n_{0}\left(\mathrm{H}_{2} \mathrm{O}\right)<$ $1.5 \times 10^{13} \mathrm{~m}^{-3}$ Killen and Ip (1999), a factor 560 smaller which gives $n_{0}\left(\mathrm{H}_{2} \mathrm{O}\right)<$ $2.7 \times 10^{10} \mathrm{~m}^{-3}$. Ne from the volatile inventory also makes a contribution to this mass group, and the published upper limit is compatible with the measurements. However, the Ne ion flux cannot be constrained well sitting on the flank of the peak at mass 23. If the exospheric Ne would have its origin in the solar wind its exospheric density at surface would be about $n_{0}(\mathrm{Ne}) \approx 3.12 \times 10^{7} \mathrm{~m}^{-3}$, about a factor 200 less than the reported upper limit of $n_{0}(\mathrm{Ne})<6 \times 10^{9} \mathrm{~m}^{-3}$, which has been used in our calculations.

With regard to the Na-group, at $m / q=22 \ldots 26$, the ion signal has about equal contributions of $\mathrm{Mg}^{+}$and $\mathrm{Na}^{+}$ions from sputtering. In addition, the thermal release of $\mathrm{Na}$ from the population that is physically adsorbed on the surface is pro- 
viding a comparable ion contribution to this mass range. The total modeled signal in this group accounts for only about $60 \%$ of the measured signal. One possibility is that the $\mathrm{Na}$ atoms are pushed to the downwind side by the photon pressure, thus improving the transport efficiency, which is not considered here. Another possibility is that the ions recorded by FIPS originate mostly from the northern hemisphere, where twice the $\mathrm{Na}$ density on the surface was found compared to the global value (Peplowski et al., 2015).

The Si-group, at $m / q=27 \ldots 33$, is indeed mostly sputtered silicon, because of the high abundance of silicon on the surface and the high photoionization rate of Si. In addition, there is a small amount of $\mathrm{Al}^{+}$ions contributing to this mass group. Given that the sputtered Si explains the mass peak at $m / q=28$ well, the abundance of the volatile species $\mathrm{N}_{2}$ has to be $n_{0}\left(\mathrm{~N}_{2}\right)<5 \times 10^{9} \mathrm{~m}^{-3}$, well below the reported upper limit of $n_{0}\left(\mathrm{~N}_{2}\right)<2.3 \times 10^{13} \mathrm{~m}^{-3}$ Killen and Ip (1999).

The group around mass 40 is dominated by contribution from sputtered $\mathrm{Ca}^{+}$ ions, the sputtered $\mathrm{K}^{+}$ion contribution is rather minor because of the small $\mathrm{K}$ abundance on Mercury's surface. $\mathrm{Ca}^{+}$ions also have been observed optically in Mercury's tail (Vervack et al., 2010). The ion contribution by the thermal desorption of the K population that is physically adsorbed on the surface is small.

Finally, based on our model we predict a small contribution of $\mathrm{Fe}^{+}$ions to be part of the magnetospheric ion population, which is outside the measurement range of FIPS.

Overall, there is a good qualitative agreement between the measured and modeled ion mass spectra. The most significant difference between the modeled and observed spectrum is at mass 32 , where the modeled sulfur peak is much smaller than the measurement. However, at mass 32 there is a spurious signal of the instrument, and it is likely that some of the counts at mass 32 are actually due to dark counts, so that agreement with modeled spectra is not necessarily expected. Analysis of cruise data in early 2009 revealed the presence of additional dark counts not identified in ground calibration, mostly in TOF channels corresponding to $\mathrm{m} / \mathrm{q}$ above $30 \mathrm{u}$ /e. A dark count removal algorithm was developed and implemented for orbital data (Gershman et al., 2013), which effectively eliminates these counts. However, this algorithm has not been implemented on data from the first flyby, as it requires the large numbers of event words available only in later observations.

The presence of the volatile species $\mathrm{O}_{2}$ and ${ }^{40} \mathrm{Ar}$ in the exosphere can make up for the difference between the model and the measurement. An $\mathrm{O}_{2}$ exospheric density at the surface of about $n_{0}\left(\mathrm{O}_{2}\right) \approx 1.6 \times 10^{11} \mathrm{~m}^{-3}$, a factor 160 less than the reported upper limit, and an Ar density of $n_{0}\left({ }^{40} \mathrm{Ar}\right) \approx 4.4 \times 10^{10} \mathrm{~m}^{-3}$, a factor 150 less than the reported upper limit, are compatible with the FIPS measurement. Although the FIPS mass spectrum is limited to $m / q=42$, we can constrain the $\mathrm{CO}_{2}$ exospheric density based on the flank of the peak at $m / q=40$ to $n_{0}\left(\mathrm{CO}_{2}\right)<$ $4.0 \times 10^{9} \mathrm{~m}^{-3}$, well below the reported upper limit of $n_{0}\left(\mathrm{CO}_{2}\right)<1.6 \times 10^{13} \mathrm{~m}^{-3}$ Killen and Ip (1999).

\section{Conclusions}

We modeled the exospheric densities for the release processes sputtering and thermal desorption in detail for the time period of the first MESSENGER flyby of Mercury. For the sputter process we calculated the ion populations from the known surface composition of Mercury, and no fitting to the FIPS measurements was applied; for thermal desorption we used reported exospheric densities from the literature, which are mostly upper limits. From the exospheric densities we calculate ion production rates, where the largest contributions to the ion flux are from the near 


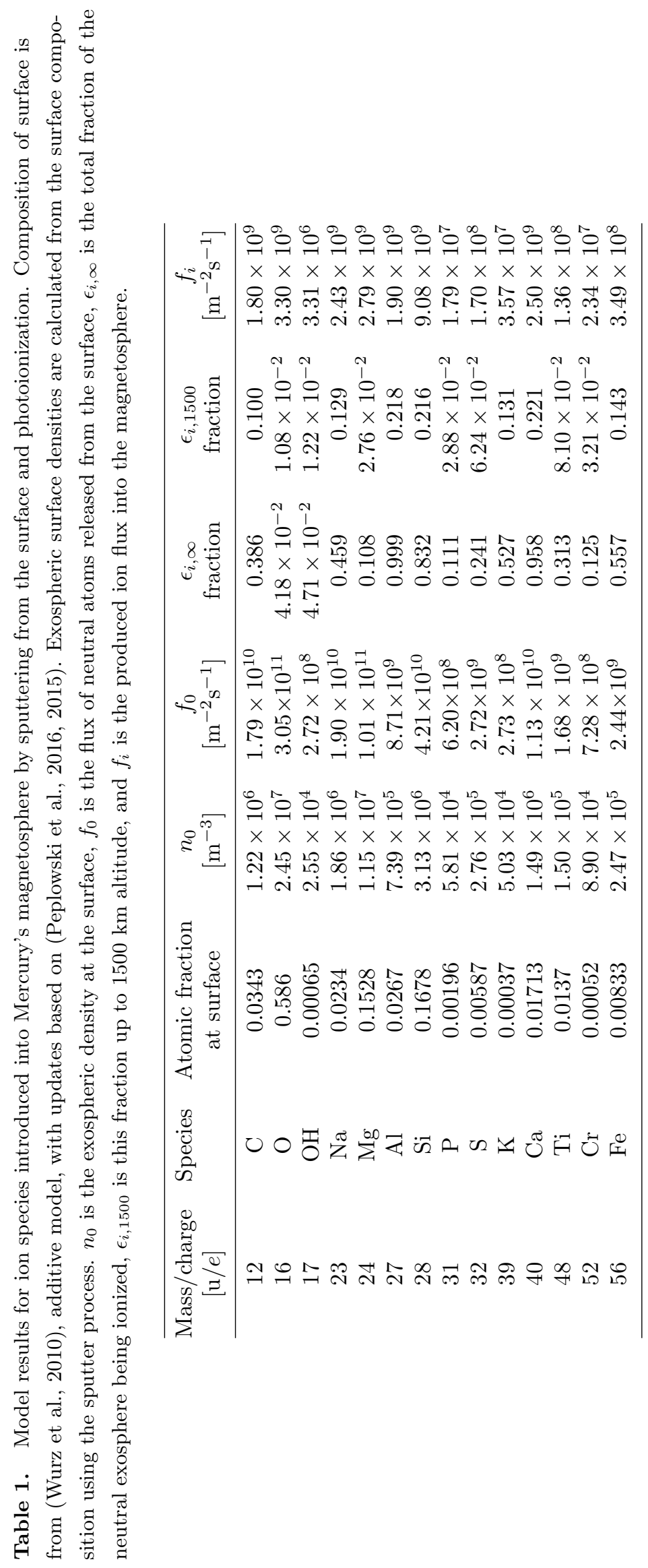




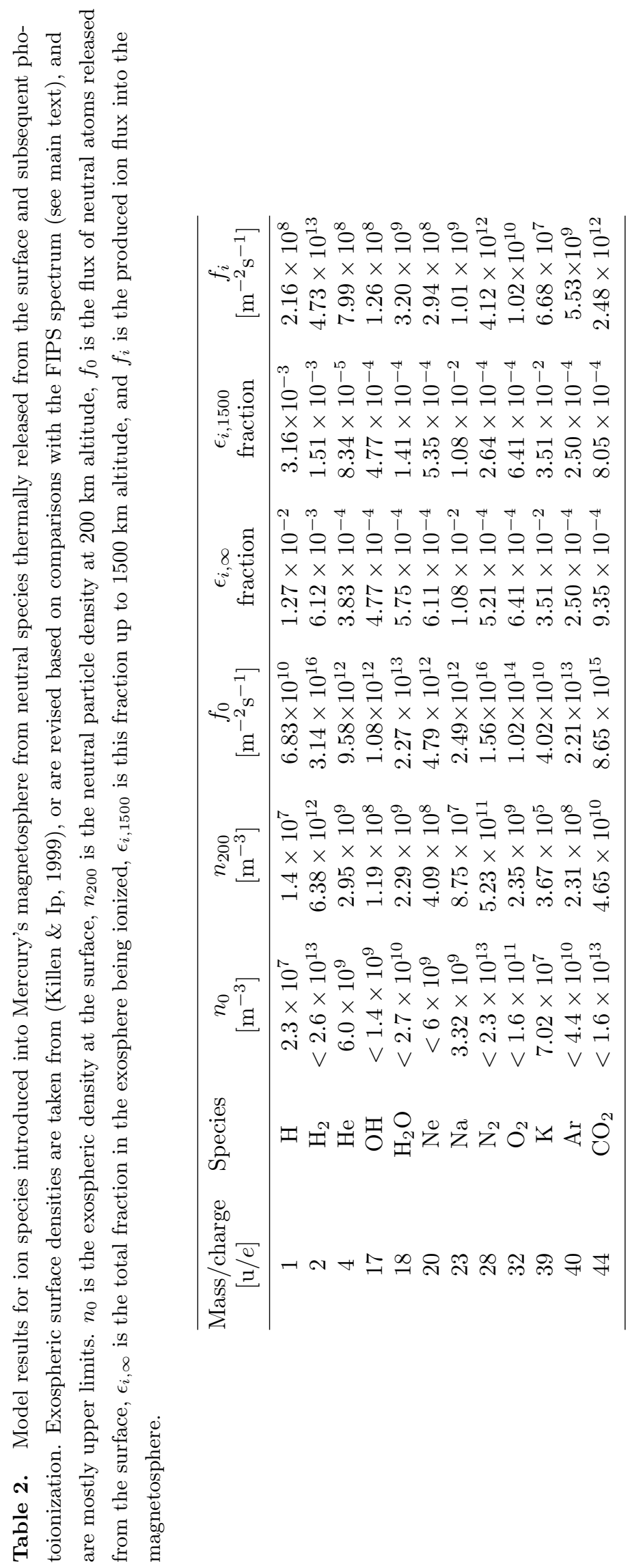


surface space. A fraction of these ions will be transported to the tail if ionization occurs when the particle is on magnetic fields lines connecting to the downwind side. From these ions we produce mass spectra that we compared with the FIPS measurements performed during the flyby.

We find good qualitative agreement between the modeled sputtered ions and the measured ion mass spectrum. Solar wind induced sputtering is the major process to contribute planetary ions to the magnetospheric ion population. In addition, thermal desorption of $\mathrm{Na}$ contributes a significant amount to the magnetospheric ion population. From the volatile species we can identify $\mathrm{He}, \mathrm{OH}, \mathrm{H}_{2} \mathrm{O}$, and $\mathrm{Ne}$ in the measured mass spectrum. However, for most of the volatile species the upper limits reported earlier have to be reduced by orders of magnitude to be compatible the the measured ion spectrum.

\section{Acknowledgments}

Data used to create Figure 3 presented in this paper can be retrieved from Vorburger (2019).

The work of P. Wurz, D. Gamborino and A. Vorburger is supported by the Swiss National Science Foundation, grant 200020-172488. J. M. Raines was supported by NASA grants NNX15AE77G and NNX15AL01G.

\section{References}

Anderson, B. J., Johnson, C. L., Korth, H., Purucker, M. E., Winslow, R. M., Slavin, J. A., ... Zurbuchen, T. H. (2011, September). The Global Magnetic Field of Mercury from MESSENGER Orbital Observations. Science, 333, 1859-1862. doi: 10.1126/science.1211001

Andrews, G. B., Zurbuchen, T. H., Mauk, B. H., Malcom, H., Fisk, L. A., Gloeckler, G., ... Raines, J. M. (2007, August). The Energetic Particle and Plasma Spectrometer Instrument on the MESSENGER Spacecraft. Space Science Rev., 131, 523-556. doi: 10.1007/s11214-007-9272-5

Delcourt, D. C. (2013, October). On the supply of heavy planetary material to the magnetotail of Mercury. Annales Geophysicae, 31, 1673-1679. doi: 10.5194/ angeo-31-1673-2013

Delcourt, D. C., Grimald, S., Leblanc, F., Berthelier, J.-J., Milillo, A., Mura, A., ... Moore, T. E. (2003, August). A quantitative model of the planetary Na+ contribution to Mercury's magnetosphere. Annales Geophysicae, 21, 17231736. doi: 10.5194/angeo-21-1723-2003

Delcourt, D. C., Moore, T. E., Orsini, S., Milillo, A., \& Sauvaud, J.-A. (2002, June). Centrifugal acceleration of ions near Mercury. Geophys. Res. Lett., 29, 1591. doi: 10.1029/2001GL013829

Gamborino, D., \& Wurz, P. (2018, September). Velocity distribution function of Na released by photons from planetary surfaces. Planet. Sp. Sc., 159, 97-104. doi: 10.1016/j.pss.2018.04.021

Gershman, D. J., Gilbert, J. A., Raines, J. M., Gloeckler, G., Tracy, P. J., \& Zurbuchen, T. H. (2013). Post-processing modeling and removal of background noise in space-based time-of-flight sensors (Unpublished doctoral dissertation). University of Michigan, MI, USA.

Kallio, E., \& Janhunen, P. (2003, September). Solar wind and magnetospheric ion impact on Mercury's surface. Geophys. Res. Lett., 30, 1877. doi: 10.1029/ 2003GL017842

Killen, R. M., Cremonese, G., Lammer, H., Orsini, S., Potter, A. E., Sprague, A. L., ... Mura, A. (2007, October). Processes that Promote and Deplete the Exosphere of Mercury. Space Science Rev., 132, 433-509. doi: 10.1007/ 
s11214-007-9232-0

Killen, R. M., \& Ip, W.-H. (1999). The surface-bounded atmospheres of Mercury and the Moon. Reviews of Geophysics, 37, 361-406. doi: 10.1029/ 1999RG900001

Lide, D. R. (Ed.). (2003). Crc handbook of chemistry and physics (84 ${ }^{\text {th }}$ Edition ed., Vols. Section 6, Fluid Properties; Vapor Pressure). CRC Press, Boca Raton, Florida, USA.

Massetti, S., Orsini, S., Milillo, A., Mura, A., De Angelis, E., Lammer, H., \& Wurz, P. (2003, December). Mapping of the cusp plasma precipitation on the surface of Mercury. Icarus, 166, 229-237. doi: 10.1016/j.icarus.2003.08.005

Peplowski, P. N., Klima, R., Lawrence, D., Ernst, C., Denevi, B., Frank, E., ... Solomon, S. C. (2016). Remote sensing evidence for an ancient carbon-bearing crust on mercury. Nature Geoscience, 9, 273-276.

Peplowski, P. N., Lawrence, D. J., Feldman, W. C., Goldsten, J. O., Bazell, D., Evans, L. G., ... Weider, S. Z. (2015, June). Geochemical terranes of Mercury's northern hemisphere as revealed by MESSENGER neutron measurements. Icarus, 253, 346-363. doi: 10.1016/j.icarus.2015.02.002

Pfleger, M., Lichtenegger, H. I. M., Wurz, P., Lammer, H., Kallio, E., Alho, M., ... Martín-Fernández, J. A. (2015, September). 3D-modeling of Mercury's solar wind sputtered surface-exosphere environment. Planet. Sp. Science, 115, 90101. doi: 10.1016/j.pss.2015.04.016

Raines, J. M., DiBraccio, G. A., Cassidy, T. A., Delcourt, D. C., Fujimoto, M., Jia, X., .. Wurz, P. (2015, October). Plasma Sources in Planetary Magnetospheres: Mercury. Space Science Rev., 192, 91-144. doi: 10.1007/ s11214-015-0193-4

Raines, J. M., Gershman, D. J., Slavin, J. A., Zurbuchen, T. H., Korth, H., Anderson, B. J., \& Solomon, S. C. (2014, August). Structure and dynamics of Mercury's magnetospheric cusp: MESSENGER measurements of protons and planetary ions. J. Geophys. Res., 119, 6587-6602. doi: 10.1002/2014JA020120

Raines, J. M., Gershman, D. J., Zurbuchen, T. H., Sarantos, M., Slavin, J. A., Gilbert, J. A., ... Solomon, S. C. (2013, April). Distribution and compositional variations of plasma ions in Mercury's space environment: The first three Mercury years of MESSENGER observations. J. Geophys. Res., 118, 1604-1619. doi: 10.1029/2012JA018073

Raines, J. M., Tracy, P., Gershman, D. J., Poh, G. K., Slavin, J. A., Zurbuchen, T., ... Solomon, S. C. (2014, December). MESSENGER's low-altitude plasma observations in Mercury's northern magnetospheric cusp. AGU Fall Meeting Abstracts, P21C-3924.

Tracy, P. J. (2016). In-situ plasma analysis of ion kinetics in the solar wind and hermean magnetosphere (Unpublished doctoral dissertation). University of Michigan, Ann Arbor, USA.

Vervack, R. J., McClintock, W. E., Killen, R. M., Sprague, A. L., Anderson, B. J., Burger, M. H., .. I Izenberg, N. R. (2010, August). Mercury's Complex Exosphere: Results from MESSENGER's Third Flyby. Science, 329, 672-675. doi: $10.1126 /$ science. 1188572

Vorburger, A. (2019, Mar). Mercury Heavy Ion Composition. osf.io/s6h9r. Open Science Framework, OSF.

Vorburger, A., \& Wurz, P. (2018, September). Europa's ice-related atmosphere: The sputter contribution. Icarus, 311, 135-145. doi: 10.1016/j.icarus.2018.03.022

Winslow, R. M., Johnson, C. L., Anderson, B. J., Korth, H., Slavin, J. A., Purucker, M. E., \& Solomon, S. C. (2012, April). Observations of Mercury's northern cusp region with MESSENGER's Magnetometer. Geophys. Res. Lett., 39, L08112. doi: 10.1029/2012GL051472

Wurz, P. (1999). Heavy ions in the solar wind: Results from soho/celias/mtof (Habilitation Thesis). Bern, Switzerland: University of Bern. 
Wurz, P., Abplanalp, D., Tulej, M., \& Lammer, H. (2012, December). A neutral gas mass spectrometer for the investigation of lunar volatiles. Planet. Sp. Science, 74, 264-269. doi: 10.1016/j.pss.2012.05.016

Wurz, P., \& Lammer, H. (2003, July). Monte-Carlo simulation of Mercury's exosphere. Icarus, 164, 1-13. doi: 10.1016/S0019-1035(03)00123-4

Wurz, P., Rohner, U., Whitby, J. A., Kolb, C., Lammer, H., Dobnikar, P., \& MartínFernández, J. A. (2007, November). The lunar exosphere: The sputtering contribution. Icarus, 191, 486-496. doi: 10.1016/j.icarus.2007.04.034

Wurz, P., Whitby, J. A., Rohner, U., Martín-Fernández, J. A., Lammer, H., \& Kolb, C. (2010, October). Self-consistent modelling of Mercury's exosphere by sputtering, micro-meteorite impact and photon-stimulated desorption. Planet. Sp. Science, 58, 1599-1616. doi: 10.1016/j.pss.2010.08.003

Zurbuchen, T. H., Raines, J. M., Gloeckler, G., Krimigis, S. M., Slavin, J. A., Koehn, P. L., ... Solomon, S. C. (2008, July). MESSENGER Observations of the Composition of Mercury's Ionized Exosphere and Plasma Environment. Science, 321, 90-92. doi: 10.1126/science.1159314 


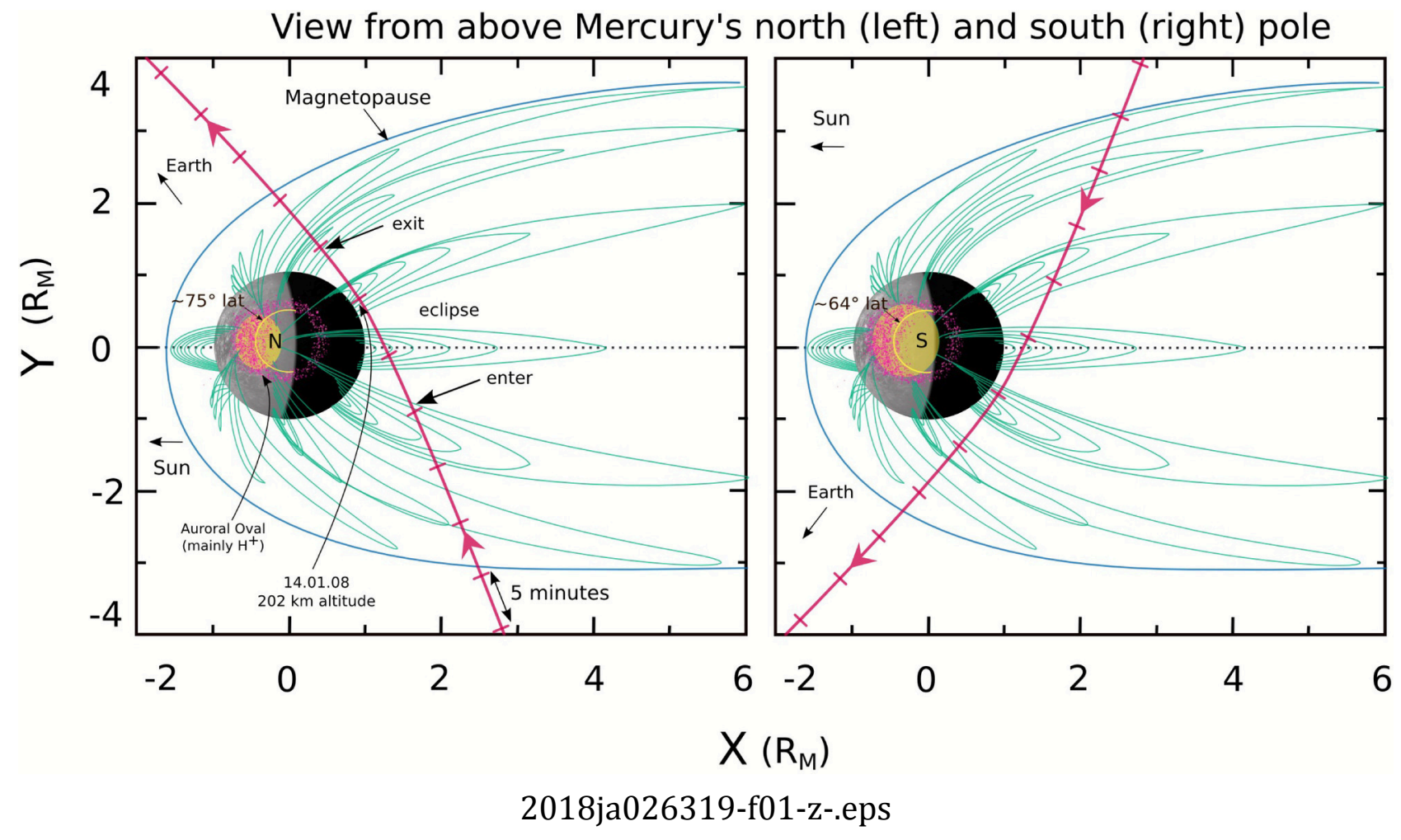

This article is protected by copyright. All rights reserved. 


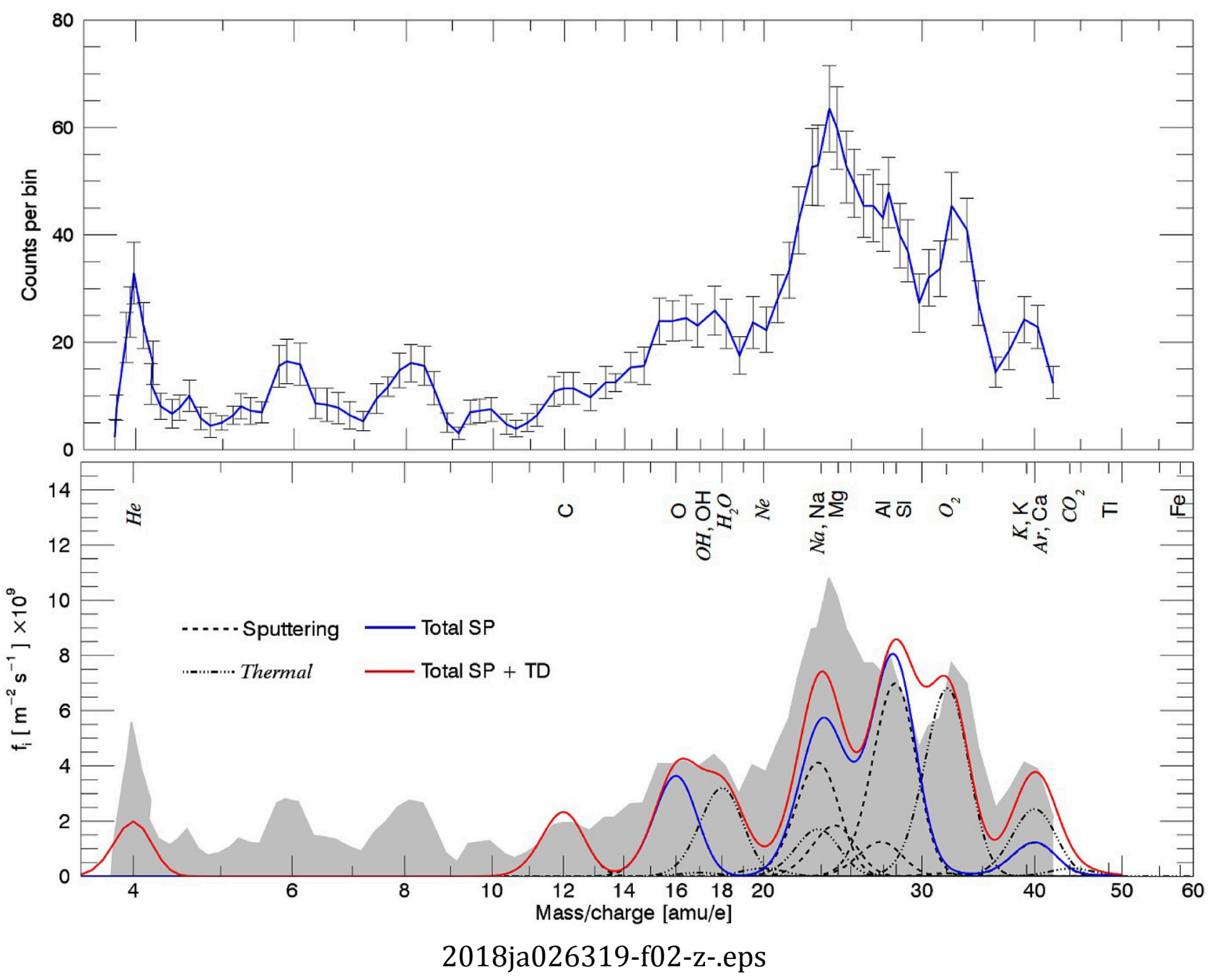

This article is protected by copyright. All rights reserved. 

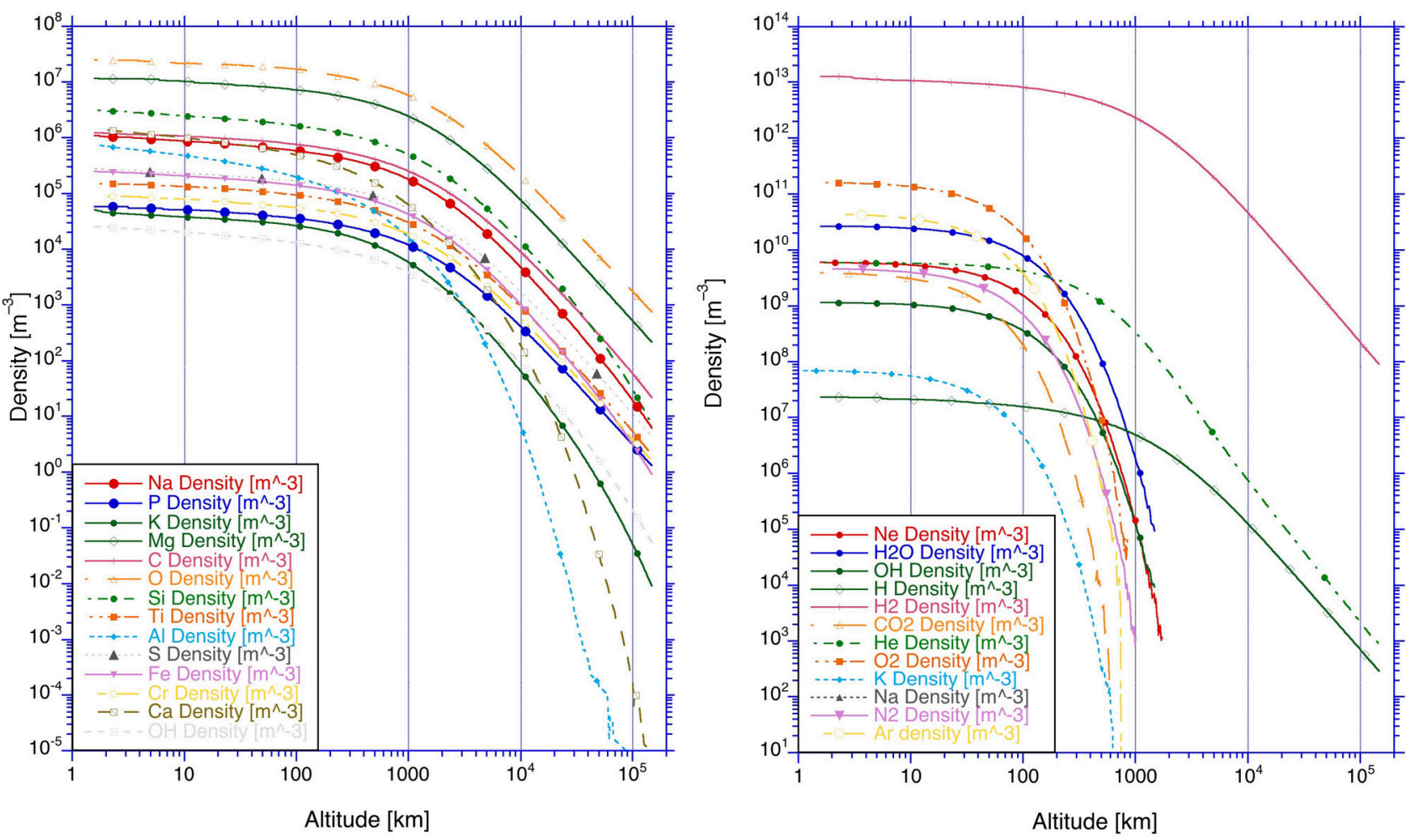

2018ja026319-f03-z-.eps 